\title{
Nanoscale
}

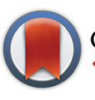

CrossMark

\&lick for updates

Cite this: Nanoscale, 2016, 8, 11177

\section{From brittle to ductile: a structure dependent ductility of diamond nanothread $\dagger$}

\author{
Haifei Zhan, ${ }^{\mathrm{a}, \mathrm{b}, \mathrm{c}}$ Gang Zhang, ${ }^{\text {b }}$ Vincent B. C. Tan, ${ }^{\mathrm{c}}$ Yuan Cheng, ${ }^{\mathrm{b}}$ John M. Bell, ${ }^{a}$ \\ Yong-Wei Zhang ${ }^{b}$ and Yuantong Gu*a
}

\begin{abstract}
As a potential building block for the next generation of devices/multifunctional materials that are spreading in almost every technology sector, one-dimensional (1D) carbon nanomaterial has received intensive research interests. Recently, a new ultra-thin diamond nanothread (DNT) has joined this palette, which is a 1D structure with poly-benzene sections connected by Stone-Wales (SW) transformation defects. Using large-scale molecular dynamics simulations, we found that this $\mathrm{sp}^{3}$ bonded DNT can transition from brittle to ductile behaviour by varying the length of the poly-benzene sections, suggesting that DNT possesses entirely different mechanical responses than other 1D carbon allotropes. Analogously, the SW defects behave like a grain boundary that interrupts the consistency of the poly-benzene sections. For a DNT with a fixed length, the yield strength fluctuates in the vicinity of a certain value and is independent of the "grain size". On the other hand, both yield strength and yield strain show a clear dependence on the total length of DNT, which is due to the fact that the failure of the DNT is dominated by the SW defects. Its highly tunable ductility together with its ultra-light density and high Young's modulus makes diamond nanothread ideal for the creation of extremely strong three-dimensional nano-architectures.
\end{abstract}

Received 23rd March 2016

Accepted 9th May 2016

DOI: $10.1039 / c 6 n r 02414 a$

www.rsc.org/nanoscale
Recently, a new 1D $\mathrm{sp}^{3}$ carbon nanomaterial has been reported, which is synthesized through the solid-state reaction of benzene under high-pressure, termed as diamond nanothread (DNT). ${ }^{12}$ On the one hand, the DNT is a close-packed $\mathrm{sp}^{3}$-bonded carbon structure, with carbon atoms arranged in a diamond-like tetrahedral motif (see Fig. 1), similar to the diamond nanowire/nanorod. On the other hand, the DNT can be regarded as hydrogenated $(3,0)$ CNTs connected with Stone-Wales (SW) transformation defects (see inset of Fig. 1). ${ }^{13}$ Meanwhile, unlike CNTs, the existence of SW transformation defects interrupts the central hollow of the structure. Thus, a fundamental understanding of how the mechanical properties of the DNT differ from its counterpart (i.e., CNT and diamond nanowire) is of great interest.

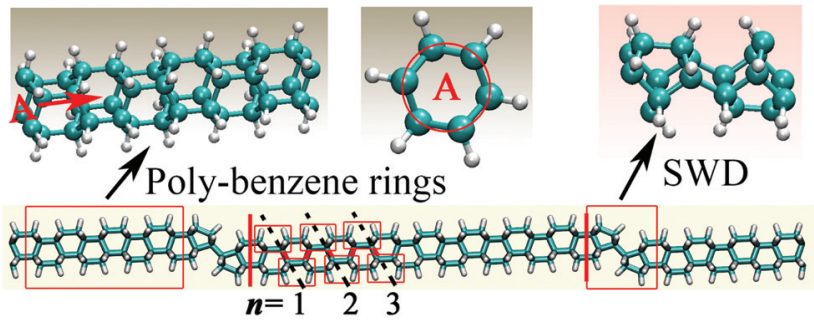

Fig. 1 The atomic configurations of a segment of DNT; insets show the structural representation of the poly-benzene rings and the StoneWales defect (SWD).

\footnotetext{
${ }^{a}$ School of Chemistry, Physics and Mechanical Engineering, Queensland University of Technology (QUT), Brisbane, QLD 4001, Australia. E-mail: yuantong.gu@qut.edu.au ${ }^{b}$ Institute of High Performance Computing, Agency for Science, Technology and Research, 1 Fusionopolis Way, Singapore 138632, Singapore.

E-mail: zhangg@ihpc.a-star.edu.sg

${ }^{c}$ Department of Mechanical Engineering, National University of Singapore, 9 Engineering Drive 1, Singapore 117576, Singapore

$\dagger$ Electronic supplementary information (ESI) available: The discussion on the influence from cut-off distance of the AIREBO potential on DNT, CNT and graphene; the comparison of the mechanical properties between DNTs with evenly and unevenly distributed SW defects; the simulation settings and results for a confined region; and the fitting results using the serial spring model. See DOI: 10.1039/c6nr02414a
} 
Previous studies have shown that the carbon nanotubes exhibit outstanding mechanical strength. For example, experimentally measured tensile Young's modulus for SWNTs ranges from $320 \mathrm{GPa}$ to $1.47 \mathrm{TPa}$ with the breaking strengths ranging from 13 to $52 \mathrm{GPa}$, and a breaking strain of up to $5.3 \% .{ }^{14}$ Whereas, a first-principles calculation suggests that the diamond nanowire has a low Young's modulus ranging from $\sim 40$ to $290 \mathrm{GPa}^{15}$ Unfortunately, most diamond nanostructures are brittle and easily fail under tensile load, which limits their applications as a building block in the nanoscale. Very recently, a preliminary study ${ }^{16}$ has shown that DNT has excellent mechanical properties, namely, a high stiffness of about $850 \mathrm{GPa}$, and a large bending rigidity of about $5.35 \times$ $10^{-28} \mathrm{~N} \mathrm{~m}^{2}$. A few questions arise promptly: what is the ductility of DNT? How does the sample length affect the mechanical properties? How does its internal structure affect the mechanical properties? Clearly, answers to these questions are crucial for further technological explorations. ${ }^{17-20}$ To this end, in this work, we explore the mechanical characteristics of DNTs through investigations of the length-dependency and structural-influence on their mechanical properties. From large-scale molecular dynamics simulations, we found that the DNT can transition from a brittle to a ductile behaviour, which is enabled by the ductile characteristic of the constituent SW transformation defects. This phenomenon has not been observed in other diamond nanostructures. It is worth noting that different from the bulk material, the diamond nanothread is an ultra-thin nanomaterial, which simply cannot undergo traditional plastic deformation, e.g., dislocations. Therefore, our discussions regarding the "brittle" or "ductile" characteristic are based on the context of the stress-strain curve.

\section{Computational methods}

The mechanical behaviors of DNTs were acquired based on a series of tensile tests performed using large-scale molecular dynamics (MD) simulations. To initiate the simulation, the widely used adaptive intermolecular reactive empirical bond order (AIREBO) potential was employed to describe the $\mathrm{C}-\mathrm{C}$ and $\mathrm{C}-\mathrm{H}$ atomic interactions. ${ }^{21}$ This potential has been shown to represent the binding energy and elastic properties of carbon materials well. It should be noticed that the AIREBO potential usually suffers from a non-physical high tensile stress which originates from the fixed switching function. ${ }^{22}$ To overcome this problematic issue, the cut-off distance is usually extended far from the original value of $1.7 \AA$ to $\sim 1.9-2.0 \AA^{23-28}$ For the DNT structure, our calculations suggest that a cut-off distance between 1.94-1.95 $\AA$ for AIREBO potential would result in a comparable yield strain with that obtained from the reactive force filed (ReaxFF) ${ }^{29}$ (see discussions in ESI $\dagger$ ). Thus, a cut-off distance of $1.945 \AA$ is adopted in all the simulations except further declaration.

The DNT structures were firstly optimized by the conjugate gradient minimization method and then equilibrated using Nosé-Hoover thermostat ${ }^{30,31}$ for 2 ns. Periodic boundary con- ditions were applied along the length direction during the relaxation process. To limit the influence from the thermal fluctuations, a low temperature of $50 \mathrm{~K}$ was adopted. The tensile testing was achieved by applying a constant strain rate (namely, $10^{-7} \mathrm{fs}^{-1}$ ) to the fully relaxed models, while keeping the periodic boundary conditions along the length direction. The simulation was continued until the failure of the DNT. A small time step of 0.5 fs was used for all above calculations with all the MD simulations being performed using the software package LAMMPS. $^{32}$

During the tensile simulation, the commonly used virial stress was calculated, which is defined as $^{33}$

$$
\prod^{\alpha \beta}=\frac{1}{\Omega}\left\{-\sum_{i} m_{i} v_{i}^{\alpha} v_{i}^{\beta}+\frac{1}{2} \sum_{i} \sum_{j \neq i} F_{i j}^{\alpha} r_{i j}^{\beta}\right\}
$$

Here, $\Omega$ is the volume of the system; $m_{i}$ and $v_{i}$ are the mass and velocity of atom $i ; F_{i j}$ and $r_{i j}$ are the force and distance between atoms $i$ and $j$; and the indices $\alpha$ and $\beta$ represent the Cartesian components. Considering that the DNT's analogue, $(3,0)$ CNT has a diameter of $2.35 \AA$, smaller than the graphite interlayer distance (namely, $3.52 \AA$ ), we adopted a solid cylinder to approximate the DNT's volume. The approximate distance between exterior surface hydrogens (i.e., $\sim 0.5 \mathrm{~nm}$ ) was adopted as the diameter of the cylinder following Roman et al. ${ }^{16}$ Further, the atomic virial stress was estimated according to eqn (1) as

$$
\pi_{i}^{\alpha \beta}=\frac{1}{\bar{\omega}_{i}}\left\{-m_{i} v_{i}^{\alpha} v_{i}^{\beta}+\frac{1}{2} \sum_{j \neq i} F_{i j}^{\alpha} r_{i j}^{\beta}\right\}
$$

where $\bar{\omega}_{i}$ represents the effective volume of atom $i$ and $\Omega=\Sigma \bar{\omega}_{i}$. With the obtained overall stress, the yield strain is defined as the strain threshold value where the stress shows abrupt reduction and the structure starts to fail. The corresponding stress is designated as the yield strength. For comparison purposes, the effective Young's modulus of the DNT is extracted from the stress-strain curve using linear regression. Based on the assumption of linear elasticity, the initial linear regime has been selected with a strain of up to $3 \%$. Such an approach has been widely applied to evaluate the mechanical properties of nanomaterials, and validated by earlier studies. ${ }^{16,34-36}$

\section{Results and discussion}

The diamond nanothread (DNT) models were established based on recent experimental observations and first-principles calculations. ${ }^{12}$ As illustrated in Fig. 1, the DNT contains two different sections, including the Stone-Wales (SW) transformation defects and poly-benzene rings. Of note, the polybenzene rings here are equivalent to the hydrogenated $(3,0)$ carbon nanotubes. Also, the SW transformation defects here represents the defective structure resulting from the $90^{\circ}$ rotation of a $\mathrm{C}-\mathrm{C}$ dimer, which is different from the commonly discussed pentagon-heptagon pair in CNTs or graphene. For simplicity of discussion, we emphasized the evenly distributed 
SW defects on the DNT structures, and a DNT unit cell with $n$ poly-benzene rings between two adjacent SW defects is denoted by DNT- $n$. In a recent work based on first principle calculations, ${ }^{37}$ several different $\mathrm{sp}^{3}$ nanothreads were predicted theoretically. The structures we focused on are the energetically favorable ones. It is worth mentioning that adding one SW defect to the structure will increase the total system energy by $\sim 12 \mathrm{kcal} \mathrm{mol}^{-1} .{ }^{38}$ With this consideration, even the DNT with the highest defect density (DNT-2) examined in this work is an energetically favorable structure.

\section{Length dependency}

Firstly, we investigate the length dependency of the DNT's tensile properties by examining three groups of samples that are constructed by repeating DNT-8, DNT-14 and DNT-20 units, respectively. Fig. $2 \mathrm{a}$ and b show a clear correlation between the yield strain/strength and the DNT sample length. In detail, the yield strain exhibits a relatively sharp reduction in the region when $L$ is smaller than $\sim 30 \mathrm{~nm}$, and then saturates to a certain value. Such a changing profile is found uniformly in all three groups. From Fig. 2a, the DNT comprised of shorter poly-benzene units tends to saturate to a higher yield strain. For example, with $L>40 \mathrm{~nm}$, the averaged yield strain for DNT-8 is $9.0 \pm 0.3 \%$, which is about $15 \%$ larger than that of the DNT-14 (about $7.8 \pm 0.3 \%$ ).

In comparison, the yield strength shows a similar changing pattern as presented in Fig. 2b. For instance, the yield strength experienced more than $25 \%$ reduction (from $\sim 75 \mathrm{GPa}$ to $\sim 56$ $\mathrm{GPa}$ ) for the DNT-14 when the sample length increases from $\sim 13 \mathrm{~nm}$ to $26 \mathrm{~nm}$. Afterward, it fluctuates around $56 \mathrm{GPa}$. Unlike the yield strain, the yield strength for all considered
DNTs saturates to a similar value (around $56 \mathrm{GPa}$ ), and exhibits a relation independent of the constituent units for the investigated length scope (from 13-92 nm). Recalling the morphology of the DNT (Fig. 1), the SW defect is analogous to the grain boundary and the constituent unit (poly-benzene) length is equivalent to the grain size. In other words, the yield strength of the DNT remains constant with decreasing grain size (i.e., the poly-benzene length). Further evidence for such a relationship was found when we assessed the structural influence on the mechanical properties of the DNT as discussed in the following section. It is worth noting that the strain rate would also influence the yield strength/strain. To assess such impacts, a $24 \mathrm{~nm}$ DNT-17 was considered. Tested strain rates included $1 \times 10^{-6}, 5 \times 10^{-7}, 1 \times 10^{-7}, 5 \times 10^{-8}$, and $1 \times 10^{-8}$ $\mathrm{fs}^{-1}$, as shown in the ESI (Fig. S4 $\dagger$ ). We find that the DNT exhibits different yield strength/strain at different strain rates, and higher strain rate leads to larger yield strength/strain. Specifically, the yield strength/strain shows a slight difference for extremely low strain rate (less than $1 \times 10^{-7}$ ). In-detail analysis shows that although the DNT exhibits a different yield strength/ strain, the deformation mechanisms are the same, i.e., stress concentration at the locations of SW transformation defect and the failure originating from these locations. These results signify that the strain rate $\left(10^{-7} \mathrm{fs}^{-1}\right)$ considered in this work exerts ignorable impacts on the tensile behaviour of the studied DNT, and it is suitable for the purpose of this investigation.

To explain the length dependency of the yield strain/ strength, we inspected the deformation processes of the DNT. Fig. 2d shows the virial atomic stress distribution along the length direction at the strain of $4.6 \%$. It is clear that the SW defect regions are subjected to higher stress during tensile
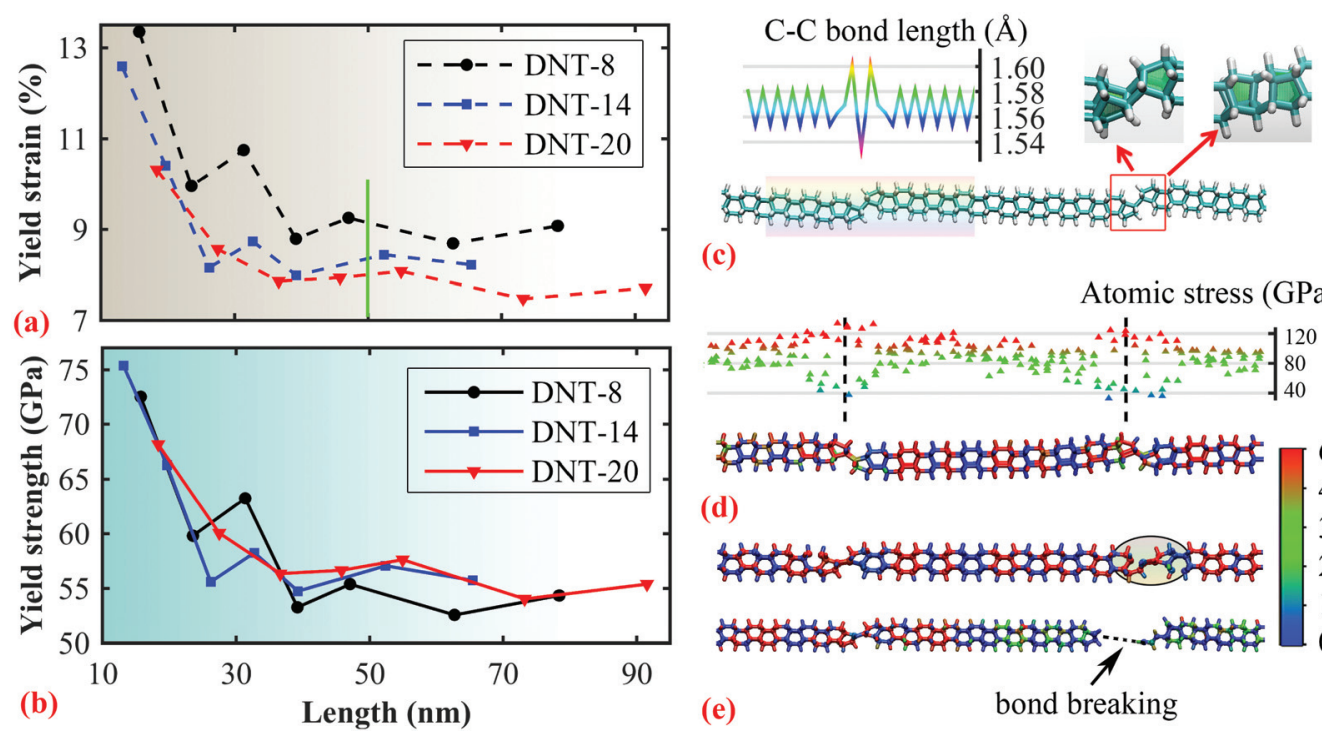

(c)

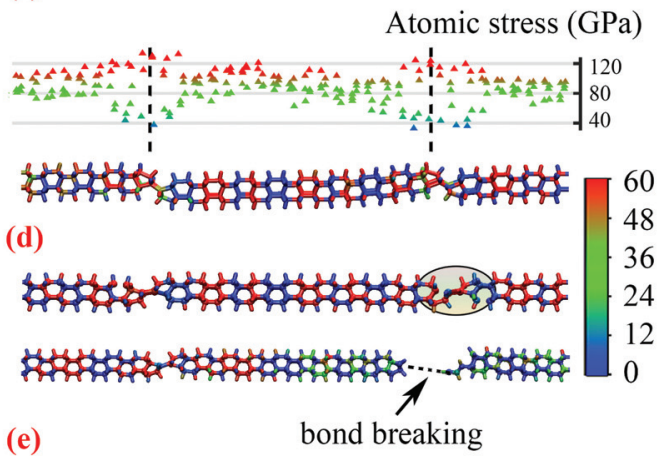

Fig. 2 (a) Estimated yield strain; and (b) yield strength of the DNT constructed by DNT-8, DNT-14 and DNT-20 units. (c) The C-C bond length distribution in the DNT, insets show the pentagons and hexagon that realized the SW defect; (d) the virial atomic stress distribution along the length direction at the strain of $4.6 \%$, which clearly shows the stress concentration at the SW defect region (only carbon atom stress is presented); (e) the bond breaking configuration at the pentagon (upper, strain of $11.2 \%$ ), which eventually initiates the failure of the DNT from the SW defect (lower, strain of $13.4 \%)$. 


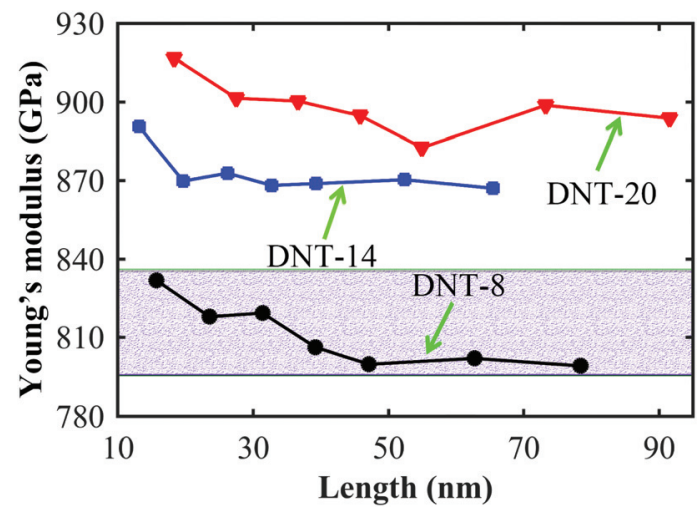

Fig. 3 Comparisons of Young's modulus extracted from different DNTs (constructed from DNT-8, DNT-14 and DNT-20 units) with varying sample length.

deformation, i.e., stress concentration. This observation is attributed to the initial bond length distribution as plotted in Fig. 2c, from which a much longer $\mathrm{C}-\mathrm{C}$ bond in the pentagons (comprising the SW defect) are observed. Thus, the SW defect regions experience a higher atomic stress after stretching. On the other hand, such a stress concentration also dominates the failure mode of the DNT. From Fig. 2e, initial bonding breaking is found in the pentagons, which eventually leads to the failure of the whole structure with increasing elongation. Such a deformation mode has been observed uniformly from the examined DNTs made from DNT-8, DNT-14 and DNT-20 units. Our simulation results demonstrate that there is a stress concentration around the SW defect region during tensile deformation. Therefore, longer DNTs possess more SW defects, meaning that they have more stress concentration regions and stronger local variances, and thus are easier to fail (i.e., leads to lower yield strain and yield strength). ${ }^{39}$

Surprisingly, although the yield strain/strength exhibits a clear length dependent characteristic, the estimated Young's modulus within each group is barely influenced by the sample length. From Fig. 3, the estimated Young's modulus for DNT-8 shows a slight reduction when $L$ increases from 15.7 to $78.4 \mathrm{~nm}$ (from 831.8 to $799.1 \mathrm{GPa}$ ). A similar trend is also observed for the other two groups comprised of DNT-14 and DNT-20, with the average Young's moduli being $872.5 \pm 1.2$ and $898 \pm 10.3 \mathrm{GPa}$, respectively. Evidently, although the total length has an insignificant influence on the Young's modulus of the DNTs, an apparent difference exists among the three groups. For instance, the average Young's modulus for DNT-8 is about $11 \%$ smaller compared with that of the DNT-20. This reveals a strong dependence of mechanical properties on the structure of the DNTs. This phenomenon can be explained from the perspective of the structural influence as detailed below.

\section{Structure - mechanical property correlation: from brittle to ductile}

The above discussions have shown that the DNT has a lengthdependent mechanical property. Another interesting question that is worth exploring is whether its mechanical behaviors can be tuned through the structural change, say, by varying the number of SW defects in a given sample length. To answer this, we constructed DNTs with a fixed length of about $42 \mathrm{~nm}$, and compared the tensile properties of DNTs containing different numbers of SW defects by changing the length of DNT- $n$. From Fig. $4 \mathrm{a}$, it is obvious that the DNT- $n$ with longer poly-benzene (larger $n$ ) exhibits a classical brittle behavior with a monotonically increased stress-strain curve; whereas, the DNTs with short poly-benzene sections, such as DNT-2, show a clear hardening process besides the monotonically increased portion. The most interesting feature is that the hardening process has greatly deferred the failure of the DNT. For example, the sample DNT-2 (which has 32 SW defects) with shorter polybenzene sections has a yield strain nearly twice that of its counterpart DNT-48, which is comprised of longer poly-benzene sections (2 SW defects). More strikingly, the hardening duration is found to extend gradually with the decrease of the constituent poly-benzene length, signifying an evident transition of the DNT from a brittle to a "ductile" behavior. This observation has been uniformly observed from the three examined groups of DNTs with the lengths of 24, 31, and $42 \mathrm{~nm}$. Explanations for such a novel observation are given later.

Despite the transition phenomenon, we found that the estimated yield strength fluctuates in the vicinity of a certain value (Fig. 4b). This phenomenon indicates a relationship independent of the number of SW defects, the same as that observed from Fig. 2b. The average value for the sample length of $42 \mathrm{~nm}$ is $57.4 \pm 1.9 \mathrm{GPa}$. Recalling the atomic configurations in Fig. $2 \mathrm{~d}$ and e, the underlying mechanism for such a relationship is that the failure normally happens at the SW defect region (when the maximum tolerable stress of the SW defect is reached). The fluctuations of the yield strength originate from the different stress distributions/localization variance at the locations of the SW defects, which are vulnerable to thermal perturbations. As evidenced from our simulations, different stress distribution patterns occur at the locations of SW defects (see ESI $\dagger$ ). In the current simulation we adopted a low temperature of $50 \mathrm{~K}$; a higher temperature is likely to influence the stress concentration and thus lead to different yield strength. A comprehensive investigation of such temperature-dependent mechanical behaviour of DNTs will be the focus of a future study.

Unlike the yield strength, the yield strain exhibits a general increasing relationship with the number of SW defects as illustrated in Fig. 4c (the results from sample lengths of $\sim 24$ and $31 \mathrm{~nm}$ exhibit a similar profile). The increasing trend is not contradictory with the previously observed length-dependent characteristic. Recalling Fig. 2a, if we consider a same sample length (the vertical green line), the DNT with a longer polybenzene section (i.e., less SW defects) also tends to have a smaller yield strain. Such results are on the one hand benefited from by the extended hardening process, and on the other hand, are due to the alleviated stress concentration. For a DNT with a fixed sample length, adding more SW defects will reduce the maximum stress in the concentration area at the same strain. As evidenced in Fig. $4 \mathrm{~d}$, the maximum stress 

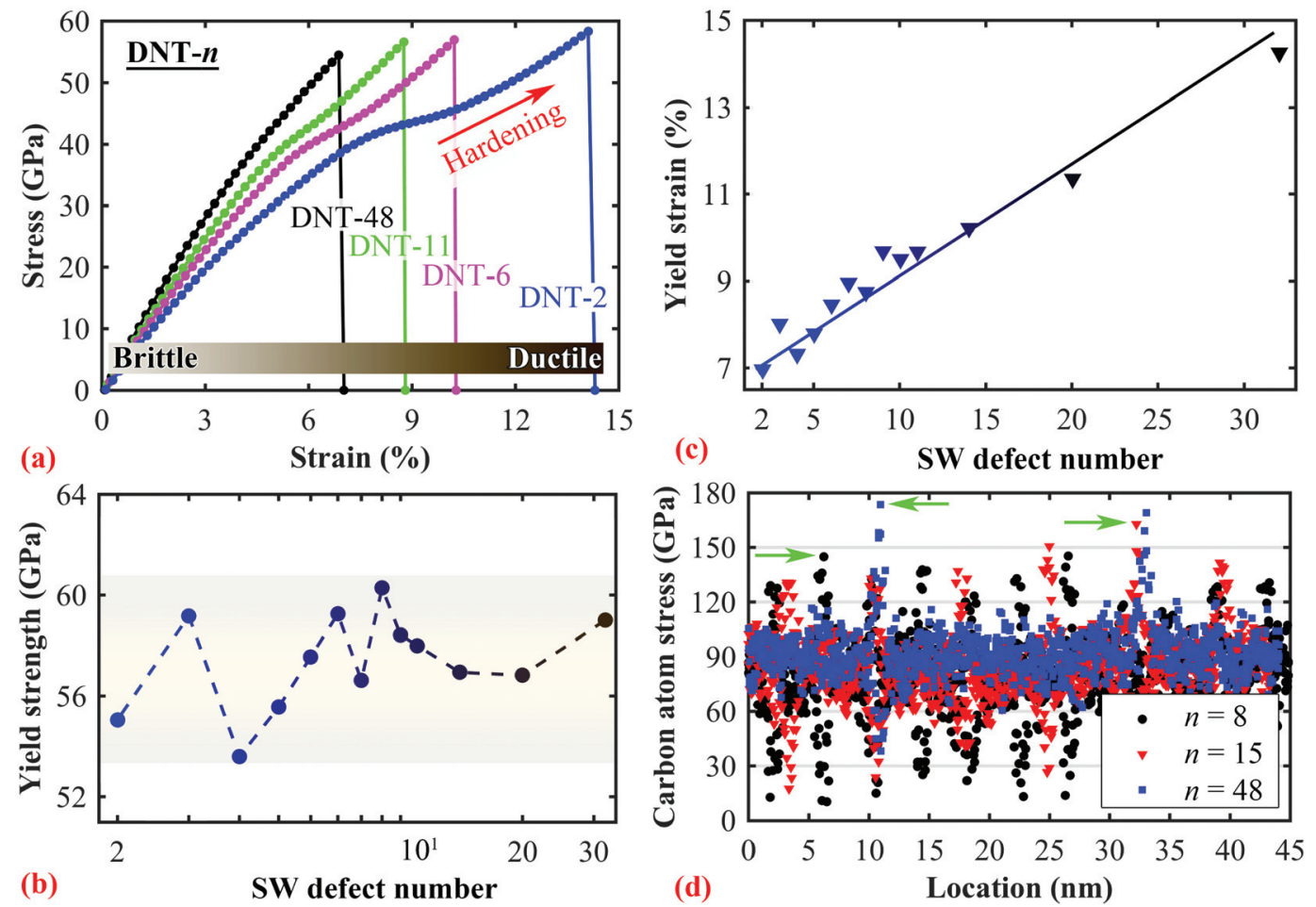

Fig. 4 Numerical results from the DNTs with a uniform sample length of $\sim 42 \mathrm{~nm}$ : (a) stress-strain curves from DNTs comprised of different constituent units; (b) the estimated yield strength, which fluctuates around a certain value, indicating a relation independent of the number of SW defects; (c) the corresponding yield strain versus the number of SW defects; (d) virial atomic stress at the strain of 5\% for DNT-8, DNT-15, DNT-48 with 11, 6, and $2 \mathrm{SW}$ defects (only carbon atom stress is presented). The green arrows highlight the maximum stress in the stress concentration regions.

in the stress concentration region is on average smaller for the structure with more SW defects. Therefore, it is reasonable to observe an increasing yield strain with the number of SW defects for a given sample length, which on the other hand affirms the increased ductility of the DNT.

\section{The ductile characteristic of the SW transformation defect}

With the above discussions, we then exploit the origins for the novel transition observed in Fig. 4a. Specifically, we track the stress-strain relation in a confined region with only polybenzene rings or the SW defect. To achieve this, we freeze the irrelevant regions and introduce a linear velocity field to stretch the targeted area (see ESI $\dagger$ ). Also, the cut-off distance was tuned to a large value of $2.0 \AA$ to ensure that the transition phenomenon does not originate from the deficiency of the AIREBO potential. As expected, we find that the confined region with only poly-benzene rings exhibits a classical brittle behavior (curve P-20), which is not affected by increasing the region length/scope (curve P-182 in Fig. 5a), signifying a brittle characteristic of the poly-benzene sections. However, for the SW defect, an extra hardening process is observed (black stress-strain curve $\underline{5}$ in Fig. $5 \mathrm{a}$ ), which endows it with a yield strain approaching $25 \%$, more than twice of that extracted from the confined region with only poly-benzene rings. These results imply the ductile characteristic of the SW defect region, which results from the initial bond breaking at the pentagon carbon rings. In-depth analyses show that only the bondstretching process was involved during the first stress increase portion (A-B in Fig. 5a). When the strain approaches 15\%, two $\mathrm{C}-\mathrm{C}$ bonds of the pentagons are found to break (see inset of Fig. 5a), which initiates the hardening process (B-C portion, refer to $\mathrm{ESI} \dagger$ for more details). The breaking of these bonds endows the SW defect region with a greatly extended deformability before fracture, which we term as a ductile characteristic. Due to the different structures, we should emphasise that the ductile characteristic discussed herein is different from the movement of 5-7 structural defects as discussed in the case of $\mathrm{CNT}^{40}$ Moreover, a fixed temperature of $50 \mathrm{~K}$ was used in our simulations. The brittle-to-ductile transitions are typically temperature dependent. Based on the unique structural property of DNT, the similar brittle-to-ductile transition is reasonably expected at room temperature. However, the comprehensive temperature impacts on the brittle-to-ductile transition deserve further studies.

Therefore, for a fixed sample length with a small number of SW defects, the DNT's mechanical behavior is dominated by the poly-benzene sections, i.e., exhibiting a brittle behavior. Vice versa, the DNT yields to a ductile behavior when more SW defects are introduced. In other words, the ductility of DNT can be controlled by altering the number of SW defects. As illustrated in Fig. 5a, by continuing to add poly-benzene rings to the two ends of the confined SW transformation defect 

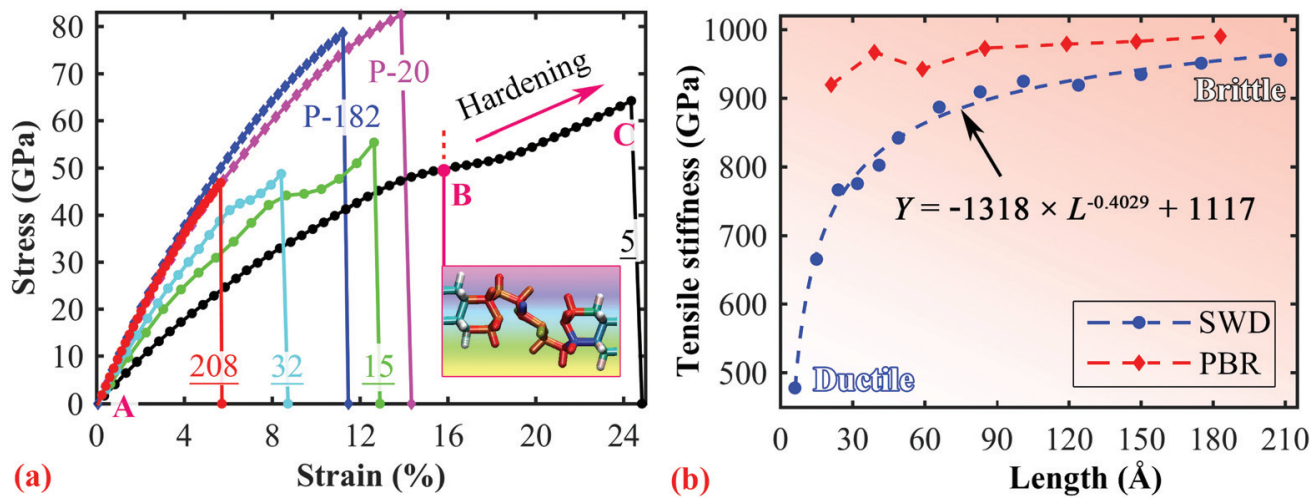

Fig. 5 (a) The stress-strain curves obtained from different confined regions with poly-benzene rings and SW defects. P-20 and P-182 denote the two confined regions containing only poly-benzene rings with a length of about 20 and $182 \AA$, respectively. The other underlined numbers represent the approximate length of the confined region with one SW transformation defect. Inset shows the atomic configurations of the SW defects at the strain of $16.1 \%$. (b) The tensile stiffness as a function of the length of the confined regions. SWD and PBR represents the confined region with SW transformation defects and poly-benzene rings, respectively. Clearly, the tensile stiffness for the purely poly-benzene rings is independent of the constrained region length.

region, its ductile characteristic is gradually suppressed, and eventually the DNT exhibits a brittle behavior (red stress-strain curve 208 in Fig. 5a). Accompanying with this transition process, the effective Young's modulus which is a placeholder for the tensile stiffness of the confined regions (blue curve) firstly experiences an exponential increase and then converges to the value extracted from the purely poly-benzene rings (red curve). As plotted in Fig. 5b, the tensile stiffness of the SW defect region (blue curve) increases almost 100\% (from $\sim 480$ to $900 \mathrm{GPa}$ ), showing the transition from a ductile characteristic to the brittle characteristic. These results suggest a highly tailorable mechanical property of the DNT endowed by its intriguing structure. In this respect, experimental efforts are expected to find a way to tune the density of SW transformation defects or the number of poly-benzene rings (grain size), perhaps via control of the benzene distributions, molecular weights or the reaction pressure. ${ }^{12}$

Of interest, we also compare the mechanical behaviour of the DNT with the serial spring model proposed by Roman et al. ${ }^{16}$ Basically, the DNT is simplified as a system connected by two types of springs, with one representing the polybenzene section (PB spring) and the other representing the SW transformation section (SW spring). Considering a constant mechanical property in both sections, the effective stiffness $E_{N}$ of DNT with $N$ SW defects can be predicted from

$$
\frac{1}{E_{N}}=\frac{N L_{\mathrm{SW}}}{E_{\mathrm{sw}} L}+\frac{\left(L-N L_{\mathrm{sw}}\right)}{E_{\mathrm{pb}} L}
$$

where $L_{\mathrm{sw}}$ and $E_{\mathrm{sw}}$ are the effective length and effective local stiffness of the SW defect region, respectively; $E_{\mathrm{pb}}$ is the effective local stiffness of the poly-benzene rings region; $N$ and $L$ are the number of SW defects and the sample length, respectively. Fitting eqn (3) with the MD results with $L_{\mathrm{sw}}, E_{\mathrm{sw}}$, and $E_{\mathrm{pb}}$ as fitting parameters, good agreement is found between the spring model and the MD values for DNTs with a smaller number of SW defects (Fig. 6). Apparently, the length

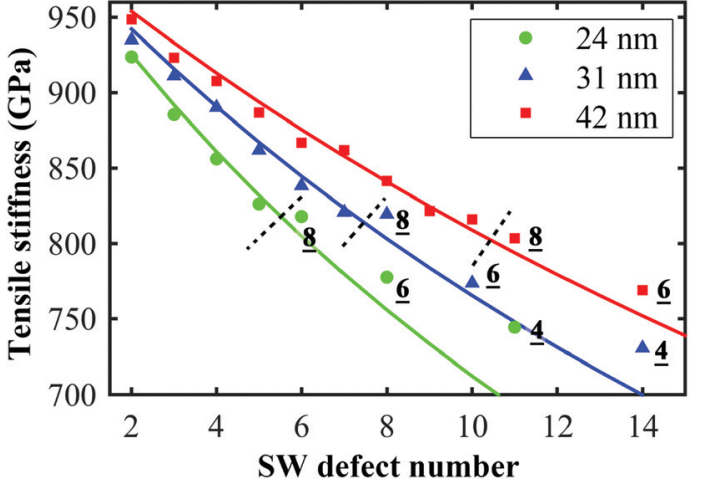

Fig. 6 Comparisons of the tensile stiffness between the spring model and the MD results. The solid lines are fitted using the data from DNT with constituent units longer than DNT-6. Obvious deviation between the spring model and the MD results is observed from DNT-8, and the shorter the poly-benzene (more number of SW defects) the larger the deviation. The underlined numbers represent the number of polybenzene rings between two adjacent SW defects in the DNT structure.

and structure dependent yield strain and strength can be well understood from the serial spring model. However, a vast difference is found for DNTs with a larger number of SW defects (see ESI $\dagger$ ), which signifies the inappropriateness of the linear spring model in describing these DNTs. Such inconsistency originates from the brittle-to-ductile transition when the DNT possesses a relatively large number of SW defects. Thus, to describe the mechanical behavior of a ductile DNT with the poly-benzene shorter than approximately $4 \mathrm{~nm}$, the spring model with nonlinear coupling should be adopted.

\section{Conclusions}

In summary, the diamond nanothread (DNT) exhibits intriguing properties that are unseen in other 1D carbon allotro- 
pies, such as diamond nanowire and CNT. Besides their excellent mechanical properties, DNTs show a brittle to a ductile transition characteristic when the length of their poly-benzene decreases (or the number of SW defects increases), suggesting a tunable mechanical property. Such a transition arises from the hardening process of the SW defect under tension. Specifically, the SW defect acts like a grain boundary that interrupts the consistency of the poly-benzene rings in the DNT structure. It is found that the yield strength of the DNT fluctuates in the vicinity of a certain value, and is independent of the "grain size" (i.e., length of poly-benzene) for a given sample length. On the other hand, both yield strength and yield strain show a considerable dependence on the total length, which is due to the fact that the failure of the DNT is dominated by the SW defect. Such intriguing properties of DNT are expected to offer appealing technological applications. Through MD simulations and theoretical analysis, we not only provide insightful understanding on the mechanical properties of the DNT, but also propose the route as a general guide for design of DNTbased devices with tunable mechanical properties. Also, the SW defect is expected to endow the DNT with intriguing thermal and electrical properties. As is evidenced from our recent work, the DNT exhibits superlattice thermal transport characteristics. ${ }^{41}$ Moreover, the highly tailorable characteristics of the structure and mechanical properties of DNTs are expected to be found in larger cross-linked systems, such as DNT-based yarn or fiber. Besides the altered individual thread strength due to the cross-links, a similar brittle/ductile transition characteristic is expected for such cross-linked systems. By tuning the distributions and density of the cross-links, the mechanical properties of DNT-based bundles can be controlled.

\section{Acknowledgements}

Support from the ARC Discovery Project (DP130102120), the Australian Endeavour Research Fellowship, and the High Performance Computer resources provided by the Queensland University of Technology, and A*STAR Computational Resource Centre (Singapore) are gratefully acknowledged.

\section{References}

1 L. Sun, J. Gong, D. Zhu, Z. Zhu and S. He, Adv. Mater., 2004, 16, 1849-1853.

2 S. Iijima, Nature, 1991, 354, 56-58.

3 A. K. Geim, Science, 2009, 324, 1530-1534.

4 J. Moser, A. Eichler, J. Güttinger, M. I. Dykman and A. Bachtold, Nat. Nanotechnol., 2014, 9, 1007-1011.

5 J. Chaste, A. Eichler, J. Moser, G. Ceballos, R. Rurali and A. Bachtold, Nat. Nanotechnol., 2012, 7, 301-304.

6 Z. Liu, S. Fang, F. Moura, J. Ding, N. Jiang, J. Di, M. Zhang, X. Lepró, D. Galvão and C. Haines, Science, 2015, 349, 400404.
7 H. Cheng, C. Hu, Y. Zhao and L. Qu, NPG Asia Mater., 2014, 6, e113.

8 T. Ghosh, Science, 2015, 349, 382-383.

9 J. Yang, M. Ma, L. Li, Y. Zhang, W. Huang and X. Dong, Nanoscale, 2014, 6, 13301-13313.

10 A. G. Slater and A. I. Cooper, Science, 2015, 348, aaa8075.

11 Y. Yu, L. Wu and J. Zhi, Angew. Chem., Int. Ed., 2014, 53, 14326-14351.

12 T. C. Fitzgibbons, M. Guthrie, E.-S. Xu, V. H. Crespi, S. K. Davidowski, G. D. Cody, N. Alem and J. V. Badding, Nat. Mater., 2015, 14, 43-47.

13 D. Stojkovic, P. Zhang and V. H. Crespi, Phys. Rev. Lett., 2001, 87, 125502.

14 M.-F. Yu, B. S. Files, S. Arepalli and R. S. Ruoff, Phys. Rev. Lett., 2000, 84, 5552.

15 J. Xue, Z. Jijun and J. Xin, Nanotechnology, 2011, 22, 405705.

16 R. E. Roman, K. Kwan and S. W. Cranford, Nano Lett., 2015, 15, 1585-1590.

17 X. W. Gu, C. N. Loynachan, Z. Wu, Y.-W. Zhang, D. J. Srolovitz and J. R. Greer, Nano Lett., 2012, 12, 6385-6392.

18 J. R. Greer, D. Jang and X. W. Gu, JOM, 2012, 64, 12411252.

19 L. R. Meza, S. Das and J. R. Greer, Science, 2014, 345, 13221326.

20 L. C. Montemayor and J. R. Greer, J. Appl. Mech., 2015, 82, 071012-071012.

21 D. W. Brenner, O. A. Shenderova, J. A. Harrison, S. J. Stuart, B. Ni and S. B. Sinnott, J. Phys.: Condens. Matter, 2002, 14, 783.

22 O. Shenderova, D. Brenner, A. Omeltchenko, X. Su and L. Yang, Phys. Rev. B: Condens. Matter, 2000, 61, 3877.

23 C. Carpenter, D. Maroudas and A. Ramasubramaniam, Appl. Phys. Lett., 2013, 103, 013102.

24 L. He, S. Guo, J. Lei, Z. Sha and Z. Liu, Carbon, 2014, 75, 124-132.

25 H. Zhao, K. Min and N. R. Aluru, Nano Lett., 2009, 9, 30123015.

26 T. Zhang, X. Li, S. Kadkhodaei and H. Gao, Nano Lett., 2012, 12, 4605-4610.

27 J. Zhao, N. Wei, Z. Fan, J.-W. Jiang and T. Rabczuk, Nanotechnology, 2013, 24, 095702.

28 H. F. Zhan, G. Zhang, J. M. Bell and Y. T. Gu, Appl. Phys. Lett., 2014, 105, 153105.

29 K. Chenoweth, A. C. van Duin and W. A. Goddard, J. Phys. Chem. A, 2008, 112, 1040-1053.

30 W. G. Hoover, Phys. Rev. A, 1985, 31, 1695-1697.

31 S. Nosé, J. Chem. Phys., 1984, 81, 511.

32 S. Plimpton, J. Comput. Phys., 1995, 117, 1-19.

33 J. Diao, K. Gall and M. L. Dunn, J. Mech. Phys. Solids, 2004, 52, 1935-1962.

34 D. Jang, X. Li, H. Gao and J. R. Greer, Nat. Nanotechnol., 2012, 7, 594-601.

35 J. Wang, C. Lu, Q. Wang, P. Xiao, F. Ke, Y. Bai, Y. Shen, X. Liao and H. Gao, Nanotechnology, 2012, 23, 025703. 
36 R. Agrawal, B. Peng, E. E. Gdoutos and H. D. Espinosa, Nano Lett., 2008, 8, 3668-3674.

37 E.-S. Xu, P. E. Lammert and V. H. Crespi, Nano Lett., 2015, 15, 5124-5130.

38 R. E. Roman, K. Kwan and S. W. Cranford, Nano Lett., 2015, 15, 1585-1590.
39 T. L. Li, H. Bei, J. R. Morris, E. P. George and Y. F. Gao, Mater. Sci. Technol., 2012, 28, 1055-1059.

40 M. B. Nardelli, B. I. Yakobson and J. Bernholc, Phys. Rev. Lett., 1998, 81, 4656.

41 H. Zhan, G. Zhang, Y. Zhang, V. B. C. Tan, J. M. Bell and Y. Gu, Carbon, 2016, 98, 232-237. 